\title{
Zur Auseinandersetzung mit postkolonialer Kritik - Eine diskursanalytische Betrachtung von Internetseiten entwicklungsbezogener Freiwilligendienste
}

\author{
Zita Hoefer \\ Universität Heidelberg | zita.hoefer@stud.uni-heidelberg.de
}

\section{Zusammenfassung:}

Weltwärts und entwicklungsbezogene Freiwilligendienste wurden in den vergangenen Jahren insbesondere aus postkolonialer Perspektive kritisiert. Wie setzen sich die Dienste mit dieser Kritik auseinander? Dieser Frage geht der folgende Beitrag im Rahmen einer diskursanalytischen Betrachtung von Internetseiten verschiedener Anbieter nach. Dabei zeigt sich, dass von der Kritik angebrachte Problematiken zwar teilweise Berücksichtigung finden, eine konkrete Auseinandersetzung mit postkolonialen Perspektiven jedoch größtenteils ausbleibt. Darüber hinaus lässt sich eine Verschiebung des Schwerpunktes in der Selbstdarstellung entwicklungsbezogener Freiwilligendienste auf die Freiwilligen konstatieren, welche wiederum neue Fragen nach der Wirkungsweise der Dienste aufwirft.

Schlagwörter: Weltwärts; Postkolonialismus; Kritische Diskursanalyse; Freiwilligendienste

\section{Summary:}

In recent years, Weltwärts and similar "volunteering for development" services have been criticized from a postcolonial perspective. How are the volunteer services dealing with this criticism? The following article examines this question drawing on critical discourse analysis by analyzing websites of separate volunteer organizations. The results indicate that the organizations partly consider the set of problems raised by postcolonial criticism. Still, a tangible contemplation of postcolonial perspectives is mostly absent. Furthermore, "volunteering for development" services shift the focus of their self-presentation to the volunteers. This, in turn, entails further questions regarding the volunteer service's modes of action and impact.

Keywords: Weltwärts; postcolonialism, critical discourse analysis, volunteering

\section{Einleitung}

Entwicklungsbezogene ${ }^{1}$ Freiwilligendienste (FWD) werden zunehmend aus postkolonialer Perspektive kritisiert. Besonders angestrebte Hilfeleistungen und positive gesellschaftliche Konsequenzen für die Partnerländer durch die Dienste werden zunehmend kritisch hinterfragt (Lewis 2006; Lough u. a. 2011; Perold u. a. 2013). Andere Arbeiten untersuchen die Auswirkungen auf die Freiwilligen selbst, da neben

1 Entwicklungsbezogene FWD unterscheiden sich von anderen internationalen FWD durch ihre Verortung im Kontext entwicklungspolitischer Aktivitäten (siehe weiterführend dazu Haas/Richter 2019). 
den angestrebten positiven Effekten für die Partnerländer auch Austausch und interkulturelles Lernen zu den Zielen der Dienste gehören (Sherraden/Lough/McBride 2008; Mangold 2012; Diprose 2012). Häufig werden Kritiken an den entwicklungsbezogene FWD unter Bezugnahme auf postkoloniale Theorien formuliert (Baillie Smith/Laurie 2011; Butcher/Smith 2010; Brown 2015), wobei die Reproduktion ungleicher globaler Machtstrukturen durch entwicklungsbezogene FWD im Vordergrund steht. Innerhalb der internationalen Debatte um die Auswirkungen der entwicklungsbezogenen FWD wurden auch deutsche Programme kritischen Analysen unterzogen (Kontzi 2015; Haas 2012). Die Einführung einer Süd-Nord-Komponente bei Weltwärts oder die Thematisierung historischer Hintergründe in Vorbereitungsseminaren können in diesem Kontext als erste positive Veränderungen in der Gestaltung von entwicklungsbezogenen FWD gesehen werden (Kontzi 2015: 234f.).

Dennoch haben sich bisher nur wenige Beiträge damit beschäftigt, ob die Kritik aus dem wissenschaftlichen Diskurs bis in die konkrete Praxis der entwicklungsbezogenen FWD vordringt (siehe dazu etwa Herrwig 2014). Dieser Aufsatz leistet einen Beitrag zu der Analyse der Praxis, in dem er Anzeichen für eine Thematisierung der Kritik in der Selbstdarstellung der entwicklungsbezogenen FWD untersucht. Dahinter steht die Annahme, dass eine Veränderung der Praxis eine Veränderung der Dienste selbst mit sich bringt, welche sich in der Selbstdarstellung widerspiegelt. Internetseiten kommt dabei eine zentrale Rolle bei der Selbstdarstellung von Organisationen und Programmen zu (Grimm/Needham 2011). Als wichtiges Informationsmedium beeinflussen sie darüber hinaus den öffentlichen Diskurs um entwicklungsbezogene FWD. Aus diesem Grund widmet sich der vorliegende Beitrag der Frage, inwiefern sich eine Auseinandersetzung mit Kritik aus postkolonialer Perspektive in der Internetpräsenz von Programmen und Organisationen widerspiegelt. Dazu wird zunächst ein Überblick über die Kritik aus postkolonialer Perspektive gegeben und die verwendete Methode der kritischen Diskursanalyse skizziert. Anschließend werden zentrale Ergebnisse vorgestellt und diskutiert.

\section{Kritik an entwicklungspolitischen Freiwilligendiensten}

Durch ihre Verortung dieser FWD-Programme in der Entwicklungszusammenarbeit (EZ), wird neben der Kritik an diesen selbst ebenfalls kurz auf Kritiken an der EZ im Allgemeinen eingegangen. Die Kritiken setzten dabei auf zwei Ebenen an: auf kultureller und auf materieller Ebene.

Innerhalb der EZ lässt sich auf kultureller Ebene eine Form des Eurozentrismus konstatieren. Bestehende Probleme im Globalen Süden sollen im Kontext der EZ durch eine Umgestaltung nach europäischem Vorbilde gelöst werden. Dies impliziert eine Überlegenheit des Globalen Nordens gegenüber dem als weniger entwickelt und rückständig geltenden Globalen Süden (Ziai 2010: 407). Auf materieller Ebene werden Abhängigkeiten unter anderem durch machtpolitische 
Bedingungen fortgeführt, die nicht selten an Unterstützungsleistungen geknüpft sind (Müller/Ziai 2015: 9). Die Kritiken an der EZ treffen auf Grund der ähnlichen Logiken ebenfalls für entwicklungsbezogene FWD zu (Brown 2015: 104). Die konkrete Kritik postkolonialer Arbeiten an entwicklungsbezogenen FWD richtet sich gegen die Stärkung des Eurozentrismus, die Reproduktion von Vorurteilen und rassistischen Stereotypen und die Fortführung von Ungleichheiten.

Der Eurozentrismus zeigt sich zum einen, ähnlich wie in der EZ, durch die inhaltliche Ausrichtung der Freiwilligendienste, welche Werte und Normen aus dem Globalen Norden in den Globalen Süden transportiert. Die zeigt beispielsweise der Aufbau von Schulen, die sich an einem westlichen Bildungsverständnis orientieren (Pluim/ Jorgenson 2012: 29). Zum anderen zeigt er sich in der Rollenbeschreibung der Freiwilligen. Sowohl die Freiwilligen selbst in ihren Erfahrungsberichten als auch die Programme und Entsenderorganisationen in ihren Programmbeschreibungen inszenieren die Freiwilligen als Entwicklungshelferinnen und -helfer. Dass die meisten Freiwilligen dabei über keine spezifischen Qualifikationen verfügen, unterstreicht das Überlegenheitsgefühl der westlichen Gesellschaften (Palacios 2010: 863).

Vorurteile und rassistische Stereotype werden ebenfalls in erster Linie in den Programmbeschreibungen (Kotzi 2015: 200) und den Erfahrungsberichten der Freiwilligen reproduziert (Müller 2015: 47). Bei Darstellungen der Einsatzländer neigen Freiwillige und Entsenderorganisationen dazu sich auf Defizite und Probleme der Empfängergesellschaften zu konzentrieren (Kontzi 2015: 212). Darüber hinaus werden kulturelle Unterschiede betont und spezifische Verhaltensweisen und Charaktereigenschaften werden mit der Zugehörigkeit zu einer bestimmten Kultur oder Nation erklärt (Müller 2015: 51). Zusätzlich betonen postkoloniale Kritiken, dass im Kontext von entwicklungsbezogenen FWD ein Bild erzeugt wird, in dem weiße Freiwillige als besonders kompetent präsentiert werden (Blum/Schäfer 2017: 5). Eine weiße Hautfarbe ist demnach weiterhin mit Macht, Wissen und Wohlstand verbunden (Bandyopadhyay/Patil 2017: 652).

Ungleichheiten werden innerhalb von entwicklungsbezogenen FWD sowohl zwischen den Ländern des Globalen Nordens und des Globalen Südens reproduziert als auch innerhalb Deutschlands. Die internationalen Ungleichheiten entstehen dabei auf der einen Seite durch fehlende Gleichberechtigung zwischen Entsende und Partnerorganisationen: Obwohl die Zusammenarbeit mittlerweile unter dem Begriff der „Partnerschaft" gefasst wird (Haas/Repenning 2018:44), liegen die Entscheidungskompetenzen über Mittelverwendung und Partizipationsmöglichkeiten mehrheitlich bei den Entsendeorganisationen (Haas/Repenning 2018: 46). Auf der anderen Seite verfestigen sich Ungleichheiten im Austausch der Freiwilligen: Obgleich Begegnung, Austausch und interkulturelles Lernen zentrale Punkte der entwicklungsbezogenen FWD darstellen, sind es überwiegend Jugendliche aus dem Globalen Norden, die einen Aufenthalt im Globalen Süden absolvieren (AKLÜH 2017: 26f). Die erlernten kulturellen Kompetenzen 
kommen folglich vermehrt den Entsendegesellschaften (Fischer/Haas 2014: 50) und den Freiwilligen aus dem Norden zu Gute (McGloin/Georgeou 2016: 413f). Hinsichtlich der Ungleichheiten innerhalb Deutschlands wird bemängelt, dass vorwiegend gutsituierte, weiße Frauen an entwicklungsbezogenen FWD teilnehmen (Sherraden u. a. 2013: 15). Menschen aus sozial schwächeren Teilen der Gesellschaft haben somit weniger Zugang zu solchen interkulturellen Erfahrungen (Kontzi 2015: 110).

Für die Betrachtung der Auseinandersetzung mit postkolonialen Kritiken ergeben sich aus den vorgestellten Punkten konkretere Fragen für die folgende Analyse: Wie werden die Rolle und die Aufgaben der Freiwilligen auf den Internetseiten dargestellt? Wie werden die Partnerländer auf den Seiten beschrieben? Wie wird auf kulturelle Aspekte Bezug genommen? Welche Informationen finden sich zur Partnerschaft zwischen den Organisationen und wer hat die Möglichkeit einen entwicklungsbezogenen FWD zu absolvieren?

\section{Methode und Sample}

Inwiefern die vorgestellte Kritik zu Veränderungen der Selbstdarstellung von Anbietern und Programmen führt, wird in dieser Arbeit mit Hilfe der kritische Diskursanalyse (KDA) nach Sigfried Jäger (2015) überprüft, die sich wiederum an dem foucaultschen Diskursverständnis orientiert (Foucault 1981). Untersucht wird hier der übergeordnete Diskurs der entwicklungsbezogenen FWD, welcher mit den Diskursen um EZ, internationale Beziehungen und interkulturelles Lernen verschränkt ist. Anschließend an die Zusammenstellung eines Materialkorpus, aus dem eine Gruppe zu untersuchender Fälle ausgewählt wird, folgen die Strukturanalyse und eine Feinanalyse eines diskurstypischen Einzelfalls (Jäger 2015).

Das zugrundeliegende Material dieser Arbeit besteht aus den Internetseiten von Organisationen und Programmen zu entwicklungsbezogenen FWD. Neben der Bedeutung für die Selbstdarstellung spricht auch die Kritik selbst für eine Betrachtung der Internetseiten: Wie in Kapitel 2 bereits gezeigt, können besonders die Darstellungen der Partnerländer und der Rolle der Freiwilligen durch die Akteure in den Entsendeländern problematisch sein. Solche Programmbeschreibungen finden sich heute überwiegend auf den Internetseiten und werden von potentiellen Teilnehmenden auch dort abgerufen (Grimm/Needham 2012).

Als Netzwerk für Organisationen von entwicklungsbezogenen FWD bietet der gemeinnützige Vereine AKLHÜ mit 206 Organisationen, die im Jahr 2016 Freiwillige entsendet haben, eine umfangreiche Auflistung von Entsendeorganisationen, welche auch für diese Untersuchung die Grundlage für die Auswahl des Materialkorpus bilden (AKLHÜ 2017: 40f). Daraus wurden vier Organisationen und ein staatliches Programm für die konkrete Analyse ausgesucht, die sich möglichst stark voneinander unterscheiden, um die Breite des Feldes abbilden zu können. Darunter ist 
die Organisation, die die meisten (AFS) und die, die die wenigsten Freiwilligen entsendet hat (Augenhöhe e.V.). Mit der Anzahl der Freiwilligen sind Unterschiede hinsichtlich des Organisationsaufwands und der finanziellen Mittel verbunden. Mit dem Roten Kreuz wurde eine Organisation gewählt, die viele Freiwillige entsendet, obwohl der inhaltliche Schwerpunkt nicht im Bereich der entwicklungsbezogenen FWD liegt. Auch das Kolpingwerk bietet entwicklungsbezogene FWD neben anderen Aktivitäten an. Besonders ist hier, dass auch sehr kurze Auslandsaufenthalte möglich sind. Darüber hinaus handelt es sich beim Kolpingwerk um eine kirchliche Organisation, womit zusätzliche Werteinstellungen verbunden sein können. Da die Mehrheit der Entsendeorganisation einen Dienst über Weltwärts anbieten und die staatliche Anbindung einen Unterschied in der institutionellen Rahmung ausmacht, wurde auch die Internetseite von Weltwärts in die Auswahl mitaufgenommen. Die ausgewählten Fälle sind in Tabelle 1 aufgelistet.

Tabelle 1: Materialkorpus

\begin{tabular}{|l|l|}
\hline Organisation/Programme & Internetseite \\
\hline AFS Interkulturelle Begegnungen e.V. & https://www.afs.de/freiwilligendienste-mit-afs \\
\hline Augenhöhe e.V. & http://augenhoehe-ev.de/ \\
\hline Deutsches Rotes Kreuz & http://www.freiwilligendienste.drk.de/startseite/ \\
\hline Kolpingwerk Deutschland gGmbH & http://www.kolping-jgd.de/ \\
\hline Weltwärts & https://www.weltwaerts.de/de/ \\
\hline
\end{tabular}

Jäger selbst versteht seine Anweisung zum praktischen Vorgehen als einen erweiterbaren Werkzeugkasten (Jäger 2015: 8). Dementsprechend wurden die Kriterien für die Strukturanalyse an das Datenmaterial angepasst. Da neben der sprachlichen Gestaltung auch die inhaltliche Bearbeitung der vorgestellten Kritikpunkte relevant ist, wurde die Feinanalyse um ein inhaltsanalytisches Verfahren mit theoriegeleiteten Codes ergänzt (Mayring/Fenzel 2014). Im Rahmen der Strukturanalysen wurden Aufbau, Gestaltung und inhaltliche Schwerpunktsetzungen der Internetseiten betrachtet und zusammengefasst. In der Feinanalyse wurden thematisch zentrale Abschnitte der Internetseiten hinsichtlich konkreter sprachlicher und bildlicher Gestaltung untersucht und mit Hilfe der Codierung Stellen, an denen Kritikpunkte thematisiert wurden, herausgearbeitet. Da eine Vielzahl von Anbieterorganisationen einen Dienst über das Programm anbietet, stand die Weltwärts-Seite im Mittelpunkt der Feinanalyse.

\section{Ergebnisse}

Auf Grundlage der Struktur- und Feinanalyse kann keine explizite Nennung der postkolonialen Theorien und ihrer Kritik auf den Internetseiten festgestellt werden. Dennoch zeigt sich in einigen Themenbereichen und Textpassagen eine Sensibilität hinsichtlich der hier vorgestellten Kritikpunkte. 
Insgesamt fällt auf, dass auf allen Internetseiten die Informationen für die Freiwilligen am umfangreichsten sind, während es wesentlich weniger Informationen zu Partnerorganisationen und Ländern gibt. Dabei stehen vor allen Dingen die Rolle der Freiwilligen und die Gestaltung des Auslandaufenthalts im Mittelpunkt. Ausgehend von den Kritiken an eurozentristischen Tendenzen von entwicklungsbezogenen FWD, wurden besonders die Rollenbeschreibungen und die Darstellung des Partnerlandes im Kontext des Auslandsaufenthalts auf der Weltwärts-Seite einer sprachlichen Feinanalyse unterzogen. Bei der Suche nach Textstellen, die auf Eurozentrismus und Hierarchisierung hinweisen, fällt auf, dass bei der Beschreibung der Tätigkeiten der „Austausch und das gemeinsame interkulturelle Lernen“ sowie die Begegnung auf „Augenhöhe“ betont werden und nicht die aktive Rolle der Freiwilligen. Es wird sprachlich deutlich gemacht, dass die Aufgabe der Freiwilligen nicht darin besteht, zu helfen, sondern sich zu engagieren, zu lernen und zu unterstützen. Wie allerdings eine Begegnung auf Augenhöhe konkret gestaltet werden kann, wird nicht ausgeführt. Trotz der Betonung des Lernaspektes und dem Verzicht auf eine direkte Ansprache des Hilfe-Begriffs, finden sich Passagen in der allgemeinen Programmbeschreibung, in denen den Freiwilligen eine besonders bedeutungsvolle Rolle zugeschrieben wird. „Somit tragen sie ihre Erfahrungen in die Gesellschaft und leisten über ihren Auslandseinsatz hinaus einen persönlichen Beitrag für eine gerechtere Welt" (Weltwärts im Überblick: Programm). An Auszügen wie diesem zeigt sich, dass die Freiwilligen zwar nicht mehr ausdrücklich als Helferinnen und Helfer gegenüber den Menschen im Empfängerland oder als Expertinnen und Experten innerhalb des Projekts inszeniert werden, ihre Rolle dennoch weiterhin positiv überhöht wird. Eine starke Orientierung an westlichen Werten kann in der Zielsetzung der Demokratieförderung gesehen werden, welche eine Überlegenheit der Demokratie gegenüber anderen Gesellschaftsformen suggeriert. Hinsichtlich der Beschreibung der Partnerländer stehen gesellschaftliche Probleme zwar nicht im Mittelpunkt, Freiwillige werden aber dennoch darauf hingewiesen, dass Standards wie fließendes Wasser in vielen Ländern nicht gegeben sind.

Die Untersuchung der Auseinandersetzung mit der Entstehung von Rassismus betrachtet neben den Internettexten auch die bildliche Gestaltung der Seiten. In den Teilbereichen, die die Anforderungen an Freiwillige vorstellen, fordert Weltwärts immer wieder zur kritischen Reflexion der eigenen Kultur und der eigenen Gewohnheiten auf. Zudem wird eine grundlegende Offenheit der Freiwilligen gefordert. Weltwärts selbst verzichtet bei Ausführungen zu den Partnerländern auf Beschreibungen, die gewisse Klischees oder Stereotype bedienen. Bei der Verwendung von Bildern achtet Weltwärts darauf, typische Motive der aktiven weißen Freiwilligen bei ihrer Arbeit zu vermeiden. Diese neutrale Bildgestaltung lässt sich allerdings nicht bei allen Organisationen feststellen. So finden sich beispielsweise auf der Internetseite von AFS gleich mehrere Bilder, die junge weiße Freiwillige zwischen afrikanischen Kindern oder beim Aufbau von Brunnen und Schulen zeigen. 
Neben der Thematisierung auf kultureller Ebene finden auch die materiellen Ungleichheiten innerhalb des Entsendelandes durchaus Beachtung. So wird aktiv versucht, die homogene Gruppe der Freiwilligen zu öffnen, was sich auf der Weltwärts-Seite in den Ausführungen zu den Anforderungen an die Freiwilligen zeigt. „Eine Beeinträchtigung oder Behinderung schließt eine Teilnahme an Weltwärts nicht aus" (Weltwärts gehen: Was wird vorausgesetzt?). Es werden überdies zusätzliche finanzielle Mittel bewilligt. Zudem wird auf eine spezielle Seite für Freiwilligendienste verwiesen, eine „ausreichende Belastungsfähigkeit“ (Weltwärts gehen: Was wird vorausgesetzt?) bleibt aber Voraussetzung. Für Menschen, denen die Finanzierung Schwierigkeiten bereitet, wird ein Flyer mit Tipps zum Spendensammeln zur Verfügung gestellt, eine zusätzliche Förderung gibt es allerdings nicht.

Um Auseinandersetzungen mit internationalen Ungleichheiten herauszuarbeiten, wurde in der Feinanalyse besonders die allgemeine Programmbeschreibung auf der Internetseite untersucht. Im Kontext der Partnerschaft steht hier die Gleichberechtigung im Mittelpunkt. Darüber hinaus sollen in der Zusammenarbeit „strukturelle Ungleichheiten, die der Kontext der Entwicklungszusammenarbeit mit sich bringt, (...) nicht ausgeblendet oder negiert, sondern kritisch reflektiert werden" (Weltwärts im Überblick). Trotz dieser postulierten Gleichberechtigung fallen an anderen Stellen der Internetseite Ungleichheiten in der Aufgabenzuständigkeit auf. So wird in den Unterseiten, die sich an die Freiwilligen richten, deutlich, dass sowohl die Auswahl der Freiwilligen als auch die Betreuung im Partnerland von den Entsendeorganisationen übernommen wird. Der übergeordnete Themenbereich der globalen Ungleichheit wird insofern beachtet, als dass Weltwärts auf seiner Seite auf eine Broschüre des bildungskritischen Vereins Glokal verweist und das Verständnis globaler Ungleichheiten explizit als Lernziel formuliert. Solche Hinweise zu globaler Ungleichheit findet sich neben Weltwärts nur auf der Seite von Augenhöhe e.V.

Insgesamt zeigt sich also, dass zumindest eine indirekte Auseinandersetzung mit Themenbereichen der Kritik stattfindet. Dies geschieht auf drei Ebenen: auf sprachlicher, auf materieller und auf Ebene der Informationsvermittlung. Auf sprachlicher Ebene spiegeln sich Diskussionen und Aushandlungsprozesse in der Verwendung und der Auslassung gewisser aufgeladener Begrifflichkeiten wider. Auf materieller Ebene ist die Bereitstellung von unterschiedlichen Ressourcen ein Weg, Problematiken durch praktisches Handeln zu begegnen. Die Tatsache, dass zusätzliche Mittel für einen spezifischen Zweck zur Verfügung gestellt werden, impliziert gleichzeitig eine Relevanz für die bereitstellende Organisation. Auch die Vermittlung von Informationen zu bestimmten Themen suggeriert, dass diese für die Organisationen von Bedeutung sind. Gleichzeitig kann jedoch angenommen werden, dass Informationen, auf die nur verwiesen wird, eine geringere Wichtigkeit zukommt als solchen, die direkt thematisiert werden. 


\section{Schlussbetrachtung}

Der vorliegende Beitrag hat die Auseinandersetzung mit postkolonialen Kritiken in den Selbstdarstellungen von Entsendeorganisationen und Programmen entwicklungsbezogener Freiwilligendienste im Internet beleuchtet und kommt dabei zu dem Schluss, dass trotz einiger Veränderungen in der sprachlichen Gestaltung eine direkte Auseinandersetzung nicht stattfindet. Darüber hinaus kann abschließend festgestellt werden, dass sich mit der Fokussierung auf den Lernaspekt und der Vermeidung einer Konzentration auf den Aspekt der Hilfeleistung der Dienste der thematische Schwerpunkt der Diskussion verschiebt. Da die Organisationen davon absehen, den Mehrwert des Engagements im Empfängerland zu verorten und die Veränderung der Lebensbedingungen vor Ort ins Zentrum des Programmes zu stellen, ergeben sich bei der Betrachtung von Freiwilligendiensten neue Fragen, wobei andere in den Hintergrund rücken. Während Kontzi (2015) in ihrer Arbeit noch folgende Passage der Weltwärts-Seite zitiert: „Lernen durch tatkräftiges Helfen“(Kontzi 2015: 110), sind Formulierungen wie diese auf der aktuellen Seite nicht mehr zu finden. Es scheint nun nicht mehr in erster Linie darum zu gehen, welche Nutzen der Dienst für das Partnerland hat, sondern stärker darum, wie er sich auf das lernende Individuum auswirkt. Die Diskussion hat sich somit von der Ebene zwischenstaatlicher Zusammenhänge auf die Ebene des Individuums verschoben. Da sich Programme nicht mehr hauptsächlich als Instrumente der EZ darstellen, ist die Notwendigkeit, sich im Kontext der Freiwilligendienste mit Kontroversen der Entwicklungspolitik zu beschäftigen, weniger offensichtlich.

Bleiben die tatsächliche Praxis und die reale Gestaltung der entwicklungsbezogenen FWD trotz der neu artikulierten Ziele unverändert, so entsteht der Eindruck, die neue Rahmung diene lediglich der Vermeidung unangenehmer Kontroversen und nicht einer Auseinandersetzung, die über die sprachliche Ebene hinausgeht. Der vorliegende Artikel weist somit nicht nur auf eine geringe Thematisierung postkolonialer Kritiken in der Selbstdarstellung hin, sondern zeigt anhand der Inhalte der Internetseiten zudem eine Diskursverschiebung.

Einen interessanten Anknüpfungspunkt könnten hier organisationssoziologische Perspektiven des Neoinstitutionalismus liefern, welche davon ausgehen, dass Organisationen Wertmaßstäbe ihrer Umwelt übernehmen, um ihre Legitimität zu sichern (Meyer/Rowan 2009). Des Weiteren kann diskutiert werden, inwieweit sich Entsendeorganisationen im Kontext ihres institutionellen Hintergrundes tatsächlich kritisch mit machtpolitischen Themen beschäftigen können. Aufgrund der überwiegend staatlichen Finanzierung bleibt fraglich, inwieweit sich Organisationen tatsächlich von der EZ abgrenzen können.

Ausgehend von den Ergebnissen dieser Arbeit zur Auseinandersetzung mit Kritik in der Selbstdarstellung könnte in einem nächsten Schritt die Untersuchung der tatsächlichen Praxis wichtige Erkenntnisse liefern. 


\section{Literaturverzeichnis}

AKLHÜ e.V. (2017): Freiwillige in internationalen Freiwilligendiensten, Bonn.

Baillie Smith, Matt und Nina Laurie (2011): International volunteering and development: Global citizenship and neoliberal professionalisation today, in: Transactions of the Institute of British Geographers, vol. 36, no. 4, S. 545-559.

Bandyopadhyay, Ranjan und Vrushali Patil (2017): The white woman's burden -the racialized, gendered politics of volunteer tourism, in: Tourism Geographies, vol. 19, no. 4, S. 644-657.

Blum, Alice und Dorothee Schäfer (2017): Volunteer work as a neocolonial practice - racism in transnational education, in: Transnational Social Review, S. 1-15.

BMZ (2014): Strategie zur Zusammenarbeit von Staat und Zivilgesellschaft in der Entwicklungspolitik der Post-2015-Welt, in: BMZ-Strategiepapier, (5). Bonn.

Brown, Eleanor Joanne (2018): Understanding and engaging with development through international Volunteering, in: Journal of International Development, vol. 30, no. 1, S. 102-117.

Butcher, Jim und Peter Smith (2010): "Making a Difference” Volunteer Tourism and Development, in: Tourism Recreation Research, vol. 35, no. 1, S 27-36.

Fischer, Jörn und Benjamin Haas (2014): Übergriffiger Staat und störrische Zivilgesellschaft? Internationale Freiwilligendienste zwischen Subsidiarität und politischer Verantwortung, in: Voluntaris, 2 Jg., Heft 1, S. 30-57.

Foucault, Michelle (1981): Archäologie des Wissens, Frankfurt am Main.

Grimm, Kerry E. und Mark D. Needham (2012): Internet promotional material and conservation volunteer tourist motivations: A case study of selecting organizations and projects, in: Tourism Management Perspectives, vol. 1, no. 1, S. 17-27.

Haas, Benjamin und Sonja Richter (2019): Weltwärts im Kontext I: Der entwicklungspolitische Freiwilligendienst im nationalen und internationalen Vergleich, in: Opusculum Nr. 123, Berlin: Maecenata Institut für Philanthropie und Zivilgesellschaft.

Haas, Benjamin (2012): Ambivalenz der Gegenseitigkeit - Reziprozitätsformen des Weltwärts - Freisilligendienstes im Spiegel der Postkolonialen Theorie, Köln.

Haas, Benjamin und Alexander Repenning (2018): Transnational knowledge in volunteering for development - A postcolonial approach to Weltwärts, in: Transnational Social Review, vol. 8, no. 1, S 34-49.

Herrwig, Chris (2014). Hollow words or actual practice? A postcolonial perspective on a project within the German ASA-Programme, in: Voluntaris, 2 Jg., Heft 1, S. 58-79.

Jäger, Siegfried (2015): Kritische Diskursanalyse - Eine Einführung, 7. Aufl., Münster.

Kontzi, Kristina (2015): Postkoloniale Perspektiven auf „wetwärts“, Baden-Baden.

Lough, Benjamin. J., Amanda Morre McBride, Margaret Sherraden und Kathleen O'Hara (2011): Capacity building contributions of short-term international volunteers, in: Journal of Community Practice, vol. 19 no. 2, S. 120-137.

Mangold, Katharina (2012): „Struggling to do the right thing“: Challenges during International volunteering, in: Third World Quarterly, vol. 33, no. 8, S. 1493-1509.

Mayring, Philipp und Thomas Fenzel (2014): Qualitative Inhaltsanalyse, in: Handbuch Methoden der empirischen Sozialforschung, hrgs. von Nina Baur, Jörg Blasius, Wiesbande, S. 543-558. 
McGloin, Colleen und Nichole Georgeou (2016): 'Looks good on your CV': The sociology of voluntourism recruitment in higher education, in: Journal of Sociology, vol. 52, no. 2, S. 403-417.

Meyer, John W. und Brain Rowan (2009): Institutionalisierte Organisationen. Formale Struktur als Mythos und Zeremonie, in: Neo-Institutionalismus in der Erziehungswissenschaft, hrgs. von: Sascha Koch, Michael Schemmann, Wiesbaden, S. 28-56.

Müller, Franziska und Aram Ziai (2015): Eurozentrismus in der Entwicklungszusammenarbeit, in: Aus Politik und Zeitgeschichte, 65 Jg., Heft 7-9, S. 8-15.

Müller, Johanna (2015): Risiken und Nebenwirkungen im Europäischen Freiwilligendienst: Eine qualitative Studie über die Reproduktion von Vorurteilen in Freiwilligenblogs, in: Voluntaris, 3 Jg., Heft 1, S. 32-61.

Palacios, Carlos M. (2010): Volunteer tourism, development and education in a postcolonial world: Conceiving global connections beyond aid, in: Journal of Sustainable Tourism, vol. 18 , no. 7 , S. $861-878$.

Perold, Helene, Lauren A. Graham, Eddy Mazembo Mavungu, Karena Cronin, Learnmore Muchemwa und Benjamin J. Lough (2013): The colonial legacy of international voluntary service, in: Community Development Journal, vol. 48, no. 2, S. 179-196.

Pluim, Gary W. J. und Shelane R. Jorgenson (2012): A reflection on the broader, systemic impacts of youth volunteer abroad programmes: A Canadian perspective, in: Intercultural Education, vol. 23, no. 1, S. 25-38.

Sherraden, Margaret S., Benjamin J. Lough und Amy C. Bopp (2013): Students serving abroad: A framework for inquiry, in: Journal of Higher Education and Outreach, vol. 17, no. 2, S. 7-42.

Sherraden, Margaret S., Benjamin J. Lough und Amanda Moore McBride (2008): Effects of international volunteering and service: Individual and institutional predictors, in: Voluntas, 19 Jg., Heft 4, S. 395-421.

Ziai, Aram (2010): Postkoloniale Perspektiven auf „Entwicklung“, in: PERIPHERIE, S. 399-426.

\section{Quellen}

AFS interkulturelle Begegnung e.V. 2018: AFS, www.afs.de (30.05.2018).

Augenhöhe e.V. (2018): Herzlich Willkommen bei Augenhöhe e.V.!, www.augenhoehe-ev.de (30.05.2018).

Deutsches Rotes Kreuz (2018): Freiwilligendienste im Ausland, www.freiwilligendienste.drk. de/freiwilligendienste-im-ausland/ (30.05.2018).

Kolping gGmbH Freiwilligendienste (2018): Was sind Freiwilligendienste?, www.kolping-jgd. de/freiwilligendienste/was-sind-freiwilligendienste (30.05.2018).

Weltwärts (2018): Weltwärts, www.weltwaerts.de/de/ (30.05.2018). 